\title{
LINEAR MAPPINGS OF OPERATOR ALGEBRAS
}

\section{B. RUSSO ${ }^{1}$}

In (6) it was shown that a linear mapping $\phi$ of one $C^{*}$-algebra $a$ with identity in to another which carries unitary operators in to unitary operators is a $C^{*}$-homomorphism followed by multiplication by the unitary operator $\phi(I)$, i.e. $\phi(A)=\phi(I) \rho(A), \phi\left(A^{*}\right)=\phi(A)^{*}$, and $\rho\left(A^{2}\right)=\rho(A)^{2}$ for each $A$ in $Q$. We continue in that spirit here, with the unitary group replaced first by an arbitrary semigroup contained in the unit sphere, then by the semigroup of regular contractions. By a $C^{*}$-algebra we shall mean a uniformly closed self-adjoint algebra of bounded linear operators on some Hilbert space, which contains the identity operator.

LEMMA 1. Let $@$ be a normed algebra containing a multiplicative semigroup $S$ with the following properties: (i) the linear span of $S$ is $\mathbb{B}$; (ii) $\sup \{\|s\|: s \in \mathcal{S}\}=K<\infty$. For $x$ in $B$, define $\|x\|_{\mathcal{S}}$ to be inf $\left\{\sum_{1}^{n}\left|a_{j}\right|\right.$ $: x=\sum_{1}^{n} a_{j} s_{j}, s_{j} \in \mathcal{S}, a_{j}$ complex, $\left.n \geqq 1\right\}$. Then $\|\cdot\|_{\mathcal{S}}$ is a normed algebra norm on $B$ such that $\|\cdot\| \leqq K\|\cdot\|_{\mathcal{S}}$. Furthermore, if $\delta$ and $\Im$ are multiplicative semigroups in the normed algebras $B$ and $\mathcal{C}$ resp., each satisfying (i) and (ii), and if $\phi$ is a linear mapping of $B$ into $\mathbb{C}$ such that $\phi(\delta) \subseteq J$, then for each $x$ in $\beta,\|\phi(x)\|\left\|_{\Im} \leqq x\right\|_{\mathcal{S}}$.

Proof. Verify.

Let $Q$ be a $C^{*}$ - algebra and let $\delta$ be a multiplicative semigroup contained in the unit sphere of $a$. Suppose that the linear span of $S$ is $Q$ and that $\|A\|_{\mathcal{S}}=\|A\|$ whenever $A$ is a regular element of $a$. For example $S$ could be the group of unitary operators, the semigroup of regular contractions, or the entire unit sphere of $a$.

Lemma 2. Let $\phi$ be a linear mapping of $Q$ into a $C^{*}$-algebra $B$ such that $\phi(I)=I$ and $\phi$ maps $S$ into the unit sphere of $B$. Then $\phi$ is a selfadjoint mapping, i.e. $\phi\left(A^{*}\right)=\phi(A)^{*}$.

Proof. We argue as in [2, Lemma 8]. Let $A$ be a self-adjoint element of $a$ of norm 1 . Then $\phi(A)=B+i C$, where $B$ and $C$ are selfadjoint elements of $B$. If $C \neq 0$, let $b$ be a positive number in the spectrum of $C$ (otherwise consider $-C$ ). Choose a positive in teger $n$ such that $\left(1+n^{2}\right)^{1 / 2}<b+n$. Then since $A+i n I$ is regular, $\|A+i n I\|$ $=\left(1+n^{2}\right)^{1 / 2}<b+n \leqq\|i C+i n I\| \leqq\|B+i(C+n I)\|=\|\phi(A+i n I)\|$

Received by the editors March 28, 1966.

1 This research was supported by a National Science Foundation grant. 
$\leqq\|A+i n I\|_{S}=\|A+i n I\|$, a contradiction. It follows that $\phi$ is a selfadjoint mapping.

Theorem 1. Let $Q$ and $B$ be $C^{*}$-algebras and let $S(r e s p$. J) be a multiplicative semigroup contained in the unit sphere of $a$ (resp. $B)$. Suppose that $\|A\|_{S}=\|A\|$ (resp. $\left.\|B\|_{\mathcal{J}}=\|B\|\right)$ whenever $A$ (resp. $\left.B\right)$ is a regular element of $Q$ (resp. $B)$. Let $\phi$ be a one-to-one linear mapping of $a$ onto

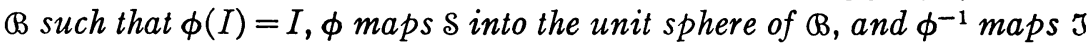
into the unit sphere of $Q$. Then $\phi$ is a $C^{*}$-isomorphism

Proof. By Lemma $2, \phi$ is a self-adjoint mapping. If $A$ is a selfadjoint element of $Q$ then $A+i I$ is regular and $\left(\|\phi(A)\|^{2}+1\right)^{1 / 2}$ $=\|\phi(A+i I)\| \leqq\|A+i I\|_{S}=\|A+i I\|=\left(\|A\|^{2}+1\right)^{1 / 2}$, so that $\|\phi(A)\|$ $\leqq A \|$. Similarly $\left\|\phi^{-1}(B)\right\| \leqq\|B\|$ for each self-adjoint element $B$ of $B$. Thus $\phi$ is an isometry of the Jordan algebra of self-adjoint elements of $Q$ onto the Jordan algebra of self-adjoint elements of $Q$ [3]. By a theorem of Kadison [3, Theorem 2], $\phi$ is a $C^{*}$-isomorphism.

The theorem shows that isometries of $C^{*}$-algebras which preserve the identity are $C^{*}$-isomorphisms [2, Theorem 7].

We now consider the semigroup $R_{1}(Q)$ of all regular contractions of a $C^{*}$-algebra $a$, i.e. the set of all invertible elements of $Q$ of norm at most one. Let $\phi$ be a linear mapping of $Q$ in to a $C^{*}$-algebra $B$ such that $\phi(I)=I$ and $\phi\left(R_{1}(Q)\right) \subseteq R_{1}(B)$. By Lemma $2, \phi$ is a self-adjoint mapping and clearly $\phi(R(Q)) \subseteq R(B)$, where $R(Q)$ denotes the group of all regular elements of the $C^{*}$-algebra $Q$.

In case $Q=\beta$ is a matrix algebra, it is known [5, Theorem 2.1] that the weaker hypothesis $\phi(R(Q)) \subseteq R(B)$ implies that $\phi$ is a Jordan homomorphism (i.e. preserves squares) followed by multiplication by a fixed regular element. We next show that this result does not generalize to arbitrary $C^{*}$-algebras except in a very special case, namely for commutative $B$.

ExAmple. Let $\propto$ be any $C^{*}$-algebra and let $\beta=M_{2}(\alpha)$ be the $C^{*}$. algebra of all 2 by 2 matrices with entries in $a$. Let $\zeta$ be any automorphism of $a$. Define a mapping $\phi$ of $Q$ into $M_{2}(Q)$ by the formula

$$
\phi(A)=\left(\begin{array}{cc}
A & A-\zeta(A) \\
0 & A
\end{array}\right), \quad(A \in Q) .
$$

Then clearly $\phi$ is a linear mapping such that $\phi(I)=I$, but it is easy to check that $\phi(R(Q)) \subseteq R\left(M_{2}(\mathbb{Q})\right)$ and that $\phi$ is not a Jordan homomorphism unless $\zeta$ is the identity automorphism.

Proposition. Let $\phi$ be a linear mapping of a $C^{*}$-algebra a into a commutative $C^{*}$-algebra $B$ such that $\phi(R(Q)) \subseteq R(\otimes)$. Then there is a 
$C^{*}$-homomorphism $\rho$ of $a$ into $B$ and an element $B$ in $R(B)$ such that $\phi(A)=B \rho(A)$ for each $A$ in $a$.

Proof. Set $\rho(A)=\phi(I)^{-1} \phi(A)$. Then $\rho(I)=I$ and $\rho(R(Q)) \subseteq R(B)$ and it suffices to show that $\rho$ is a $C^{*}$-homomorphism. Since $\rho(I)=I$, the condition $\rho(R(Q)) \subseteq R(B)$ is equivalent to $\operatorname{Sp}(\rho(A)) \subseteq \operatorname{Sp}(A)$ for each $A$ in $Q$, where $\operatorname{Sp}(A)$ denotes the spectrum of the operator $A$. If $U$ is any unitary operator in $Q$ then $\operatorname{Sp}(\rho(U))$ is a subset of the unit circle. Since $\beta$ is commutative, $\rho(U)$ is normal, hence unitary. The result follows from [6, Corollary 2].

We now return to the semigroup of regular contractions. By the remarks following Theorem 1 we may assume our mappings are selfadjoint.

LemMA 3. Let $\phi$ be a linear self-adjoint mapping of a $C^{*}$-algebra a into a $C^{*}$-algebra $@$ such that $\phi(R(Q)) \subseteq R(\Theta)$ and $\phi(I)=I$. Then (i) if $P$ is a projection in $Q$, then $\phi(P)$ is a projection in $B$; (ii) if $P$ and $Q$ are orthogonal projections in $Q$, then $\phi(P)$ and $\phi(Q)$ are orthogonal projections in B.

Proof. (i) if $P$ is a projection, then $\phi(P)$ is a self-adjoint operator with spectrum contained in the two point set $\{0,1\}$. (ii) if $U$ is a selfadjoint unitary operator in $a$ then $\phi(U)$ is self-adjoint and unitary in $B$. An operator $T$ is a projection if and only if $I-2 T$ is self-adjoint and unitary. Let $P$ and $Q$ be orthogonal projections in $Q$ and set $U=I-2 P, V=I-2 Q$. The orthogonality of $P$ and $Q$ implies that $U$ and $V$ commute. Hence $U V$ is also a self-adjoint unitary operator. Thus $\phi(U V)=I-2(\phi(P)+\phi(Q))$ is self-adjoint and unitary so that $\phi(P)+\phi(Q)$ is a projection. It follows that $\phi(P) \phi(Q)=0$.

Lemma 4. Let $\phi$ be a linear self-adjoint mapping of a commutative $C^{*}$-algebra $Q$ into a $C^{*}$-algebra $ß$ such that $\phi(R(\AA)) \subseteq R(ß)$ and $\phi(I)=I$. Then $\|\phi\|=1$.

Proof. Let $A$ be a positive element of $Q$. Then $\phi(A)$ is self-adjoint and since $\mathrm{Sp}(\phi(A)) \subseteq \mathrm{Sp}(A)$ it follows that $\phi(A)$ is positive. Thus $\phi$ is a positive mapping. By results of Stinespring [7, Theorems 1 and 4], there is a Hilbert space $K$, a *-representation $\rho$ of $a$ on $K$ and an isometry $V$ of $H$ into $K\left(B\right.$ acts on $H$ ) such that $\phi(A)=V^{*} \rho(A) V$ for all $A$ in $a$. Thus if $A \in Q$, then $\|\phi(A)\|=\left\|V^{*} \rho(A) V\right\| \leqq\|A\|$.

Recall that a von Neumann algebra is a $C^{*}$-algebra which is closed in the weak operator topology $[1$, p. 33].

THEOREM 2. Let $\phi$ be a linear mapping of a von Neumann algebra $M$ 
into a $C^{*}$-algebra $B$ such that $\phi\left(R_{1}(M)\right) \subseteq R_{1}(B)$ and $\phi(I)=I$. Then $\phi$ is a $C^{*}$-homomorphism.

Proof. As noted above, $\phi$ is self-adjoint and $\phi(R(M)) \subseteq R(B)$. Let $A$ be a self-adjoint element of $M$ of norm 1. The von Neumann algebra $M_{0}$ generated by $A$ is commutative and if $\epsilon>0$ there exist orthogonal projections $P_{1}, P_{2}, \cdots, P_{n}$ in $M_{0}$ and real numbers $r_{1}, r_{2}, \cdots, r_{n}$ such that $\left\|A-\sum_{1}^{n} r_{i} P_{i}\right\|<\epsilon[1$, p. 3]. By several applications of the preceding two lemmas and after a computation one obtains $\left\|\phi(A)^{2}-\phi\left(A^{2}\right)\right\|<2 \in(2+\epsilon)$. Since $\epsilon$ was arbitrary $\phi(A)^{2}$ $=\phi\left(A^{2}\right)$ for each self-adjoint $A$ in $M$ of norm 1. It follows trivially that $\phi$ is a $C^{*}$-homomorphism.

We note that the theorem holds with an identical proof in case $M$ is an $A W^{*}$-algebra [4].

REMARKs. 1. It is an open question as to whether Theorem 2 is true when $M$ is a $C^{*}$-algebra. Since the conclusion, i.e. $\phi(A)^{2}=\phi\left(A^{2}\right)$, need only hold for self-adjoint operators $A$, there is no loss of generality in assuming $M$ to be commutative. Then by Lemma 4 we have $\|\phi\|=1$.

2. The author believes that a solution to the following special case would shed considerable light on the problem: let $Q$ be a commutative $C^{*}$-algebra acting on a Hilbert space $H$, and let $P$ be a projection operator on $H$, say mapping $H$ onto a subspace $K$. Let $\phi$ be the mapping $\phi(A)=P A$ of $Q$ into the bounded operators on $K$. The reason for this belief is the relation of the mapping $A \rightarrow P A$ to the results of Stinespring quoted above, and to normal dilations of operators.

\section{REFERENCES}

1. J. Dixmier, Les algèbres d'operateurs dans l'espace Hilbertien, Gauthier-Villars, Paris, 1957.

2. R. V. Kadison, Isometries of operator algebras, Ann. of Math. 54 (1951), 325-338.

3. - A generalized Schwarz inequality and algebraic invariants for operator algebras, Ann. of Math. 56 (1952), 494-503.

4. I. Kaplansky, Projections in Banach algebras, Ann. of Math. 53 (1951), 235-249.

5. M. Marcus and R. Purves, Linear transformations on algebras of matrices, Canad. J. Math. 11 (1959), 383-396.

6. B. Russo and H. A. Dye, A note on unitary operators in $C^{*}$-algebras, Duke Math. J. 33 (1966), 413-416.

7. W. F. Stinespring, Positive functions on $C^{*}$-algebras, Proc. Amer. Math. Soc. 6 (1955), 211-216.

University of California, Irvine 